\title{
Audit of anticholinergic treatment in a psychiatric patient population
}

\author{
Justin H. G. Williams
}

\begin{abstract}
Aims and method An audit was performed to look at how carefully anticholinergic medication was prescribed and monitored in a population of psychiatric patients on depot medication.

Results The mean duration of prescription was 47 months and $44 \%$ of patients' prescriptions went unaltered for over three years. Evidence was lacking for rational initial anticholinergic prescription.

Clinical implications Anticholinergic treatment needs to be prescribed more rationally with regular monitoring of extra-pyramidal symptoms.
\end{abstract}

The extrapyramidal side-effects (EPS) of neuroleptics are common and potentially disabling. Anticholinergic medications such as procyclidine are widely used, accepted and are successful treatments for some of these side-effects. However, they are not without their own problems. A World Health Organization consensus statement (1990) has detailed these as: predisposing to tardive dyskinesia; causing urinary retention and paralytic ileus; impairing memory function; contributing to the development of fatal hyperthermic episodes; causing a toxic confusional state with hallucinations and paranoid delusions when consumed in excess; having a euphoriant effect which makes discontinuation difficult and; decreasing the therapeutic activity of neuroleptics. Johnstone et al (1983) found that patients with schizophrenia on procyclidine exhibited more positive psychotic symptoms than those allocated to placebo.

Patients should therefore be prescribed anticholinergic medication with caution and the dose needs to be monitored to maintain it at the minimum level. Concern that a relapse of EPS will accompany a dose reduction may deter clinicians from altering the dose, but after three months of treatment this is probably not likely to happen (Barnes, 1990; Double et ah, 1993; Bazire, 1995).

\section{Aims}

It was my clinical impression that anticholinergic medications are liberally prescribed and that dose and response tend to go unmonitored. This audit had three aims:

(a) To identify patients on long-term anticholinergic medication whether long-stay in-patients or out-patients.

(b) To look at the time elapsed since an anticholinergic prescription was last altered. After consultation with the Trust Pharmacotherapy Advisory Committee, a standard was set that a dose reduction should be attempted within three months of commencement.

(c) To find evidence from in-patients' notes of examinations performed for EPS.

\section{The study}

Letters were sent to a sample of community psychiatric nurses, hostels and wards caring for patients (18-65 years of age) on depot neuroleptics, requesting lists of patients receiving depot neuroleptics. It also asked whether or not patients were also being treated with procyclidine.

For in-patients receiving procyclidine, an examination was made of notes and drug records. For out-patients receiving procyclidine, a letter was written to their general practitioner (GP). In all cases the following questions were asked:

(d) What is the present dose of anticholinergic medication?

(e) How long has the patient been on this dose?

(f) When was the dose last changed?

(g) What is the current dose of depot medication?

\section{Findings}

The patient sample for whom initial information was returned $(n=160)$ consisted of 94 men and 66 women. Thirty-five were long-stay in-patients, 117 out-patients and eight patients resided in hostels. Ninety-three (58\%) patients were prescribed long-term procyclidine. Comparison of in-patients and out-patients found no significant 
group differences using $t$-tests, with regard to age, gender ratio, dose of procyclidine and dose of depot.

All patients in this audit prescribed anticholinergic medication received it in the form of procyclidine. One had changed from orphenadrine in the week before the study.

The likelihood of a patient being prescribed procyclidine varied significantly according to their ward ( $\chi^{2}$ test: d.f. $\left.=2, P=0.016\right)$, whereas there was not quite a significant difference according to the community psychiatric nurse that out-patients were registered with $\left(\chi^{2}\right.$ test: d.f.=7, $P=0.06$ ). Age, gender or out-patient/inpatient status had no effect (using $\chi^{2}$ tests) on the likelihood of prescription. Indeed the mean age of patients on and off procyclidine was $\mathbf{4 4 . 2}$ years and 44.7 years, respectively. The mean dose of depot was also very similar for both patient groups (785 (s.d. 940) mg v. 597 (s.d. 816) mg for patients on and off procyclidine respectively. calculated in clopixol equivalent dose, mg/week, according to Bazire, 1995).

\section{Duration of anticholinergic medication}

The mean duration of an unaltered prescription for procyclidine was 46.7 months (see Fig. 1). This was a mean of 59.7 months for out-patients and 28.6 months for in-patients.

This was usually a minimum amount of time and may have only extended as far back as the GPs' records. GPs often made a comment such as "he has never had the dose changed as long as I have known him which has been for four years", and therefore the duration recorded was 48 months.

Only $8(12 \%)$ patients met the standard of a trial of reduction in the previous three months

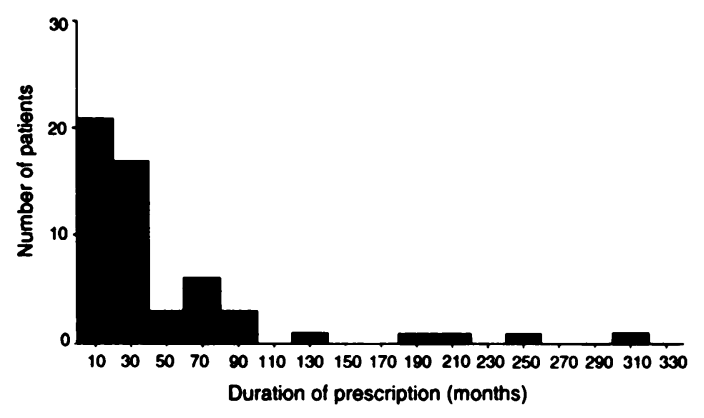

Fig. 1. Histogram showing variation in lengths of procyclidine prescriptions (mean $=47$ months, s.d.=62.18, $\mathrm{n}=55.0$ ). (six of them in-patients), with 24 (44\%) patients having had no change in their prescription for at least three years. Duration of treatment correlated with age of patient (Spearman correlation coefficient $=0.481, P<0.0001$ ), but there was no correlation between depot dose and dose of procyclidine or between these and age or treatment duration.

On an examination of the notes of in-patients, evidence for a review of anticholinergic requirement, where procyclidine or EPS were actually mentioned was found in 4/23 cases and only once was the entry "no EPS, stop procyclidine" found.

\section{Comment}

Sixty per cent of patients on depot medication were prescribed long-term procyclidine and the average duration of a prescription was nearly four years. A patient's ward appeared to affect the likelihood of a procyclidine prescription but this was unaffected by the neuroleptic dose. The picture was consistent with procyclidine being prescribed early on in an admission followed by indefinite and unmonitored continuation.

Two limitations should be noted. The audit missed those patients that reduced or stopped procyclidine by simple non-compliance as it only looked at prescription records. Second, notes of patients not on procyclidine were not examined so patients that had stopped procyclidine were not counted. However, the audit findings agreed that procyclidine is liberally prescribed and poorly monitored. This may be because procyclidine is mistakenly considered benign or not in itself therapeutic, so goes unaltered when other treatment changes are made. Alternatively, because the drug can have euphoriant effects, patients may be reluctant to accept a dose reduction and may complain of symptoms such as stiffness if this is threatened.

This audit suggests a need for more rational anticholinergic prescribing with standardised monitoring of EPS, regular attempts at dose reduction and an awareness of the issue of procyclidine misuse. Such practice should be incorporated into a continuous audit cycle.

\section{Acknowledgements}

I am especially grateful to Sarah McGeorge and the staff of the Department of Clinical Effectiveness of Norfolk Mental Health Care NHS Trust for their comments and so much assistance with data collection. I am also grateful to Duncan Double, Steve Bazire and Nina Dick for helpful comments and to the community psychiatry nurses and GPs of Norfolk for their cooperation. 


\section{References}

BARNES, T. R. E. (1990) Comment on the WHO consensus statement. British Journal of Psychiatry, 156, 413-414. BAZIRE, S. (1995) Psychotropic Drug Directory 1995, p. 42. Milton Keynes: Lundbeck.

Double. D. B., Warren, G. C., Evans, M., et al (1993) Efficacy of maintenance use of anticholinergic agents. Acta Psychiatrica Scandinavica, 88, 381-384.

JohNSTONE, E. C. Crow, T. J., Ferrier, N. I., et al (1983) Adverse effects of anticholinergic medication on positive schizophrenic symptoms. Psychological Medicine, 13. 513-527.

WORLD Health ORganization (1990) Prophylactic use of anticholinergics in patients on long-term neuroleptic treatment. A consensus statement. British Journal of Psychiatry, 156, 412.

Justin H. G. Williams, Specialist Registrar, Department of Psychiatry. University of Dundee, Ninewells Hospital and Medical School, Dundee DD1 9SY

\title{
Profile of staff grade psychiatrists
}

\author{
Aileen Blower
}

\begin{abstract}
Alms and method Staff grade psychiatrists working in Scotland were surveyed by postal questionnaire in order to determine their demographic profile, career aspirations and experience of the grade.

Results A heterogeneous group of doctors was identified, from a variety of professional backgrounds, and with a range of ambitions. Respondents generally held a favourable view of the grade, but expressed concern over percelved lack of opportunities for training, education and career progression. This is despite considerable recent efforts by the College to include non-consultant career grades in its programme for Continuing Professional Development.

Clinical implications There remains scope for imaginative development of the grade. In particular. staff grade psychiatrists might benefit from a more individualised approach to, and support for, all areas of their professional development. Such 'mentoring' may be especially beneficial for staff grades in Scotland, for whom geographical, as well as professional isolation, can be problematic.
\end{abstract}

The staff grade was introduced following the recommendation contained in the report Hospital Medical Staffing: Achieving a Balance (Department of Health and Social Security (DHSS), 1986). It was expected that doctors who were 'unable or unwilling' to become consultants would enter this grade from senior house officer level, attracted by the security and intermediate responsibility of the grade. Career advice for all junior trainees was also recommended by the report. To prevent the staff grade growing too rapidly at the expense of consultant appointments, manpower controls should ensure that the maximum number in the staff grade does not exceed $10 \%$ of the total number of consultants. Finally, in the consultation that followed, it was recognised that, although not a formal training grade, adequate and continuing education would be essential (DHSS, 1987).

Previous studies have surveyed staff grade doctors and dentists, across different specialities, within England and Wales (Standing Committee on Postgraduate Medical Education (SCOPME, 1994) and Scotland (Scottish Council for Postgraduate Medical and Dental Education (SCPME), 1996). Educational needs of staff grade psychiatrists within the South-East Thames region have also been examined (Stein, 1997). These reports have expressed concern regarding: lack of career advice; confusion over the status of the grade; need for continuing medical education; isolation in the grade; and exclusion from career progression. The larger studies also showed that the majority of staff grades in England and Wales were overseas qualified men, while, in Scotland, female UK qualifiers were over-represented.

This study focused on psychiatrists within Scotland, in order to determine if they share characteristics and views with their staff grade colleagues in all other specialities.

\section{The study}

In Spring 1997, a semi-structured questionnaire was posted to all doctors currently serving in a staff grade psychiatry post within Scotland. Staff grade colleagues assisted in compiling the 